\title{
DERIVED SETS AND THEIR COMPLEMENTS
}

\author{
BY S. T. SANDERS, JR.
}

1. Introduction. Denoting by $g A$ the derived set of a set $A$ in a general topological space* $S$, and by $c A$ the complement $S-A$ of $A$, we consider the family of sets $\phi A$, where $\phi$ is a product involving the operator $c$ and operators of the form $g^{\alpha}, \alpha$ being an ordinal, finite or transfinite. Important examples of operators are $c g, g c, g^{2} c, c g c$. The following discussion is based on the assumption of the distributive and closure properties:

I.

$$
g(A+B)=g A+g B,
$$

II.

$$
g^{2} A \subset g A \text {. }
$$

In $\$ \$ 2-5$ eight elementary sets are defined, from which a $c a$ nonical system of sets is obtained. This canonical system is sufficient for the representation of all sets of the form $\phi A$ and the finite sums and products $\sum \phi A, \Pi_{\phi} A$, and $\sum \prod_{\phi} A$, with the exception of certain subsets of the derived set of the isolated points of the space $S$. In $\S 6$ specializations of the general theory are given to spaces possessing either or both of the properties: (a) self density, (b) $g 0=0$. Under restrictions (a) and (b), the basic set of inclusions (10), which is fundamental for the discussion of $\S \S 4$ and 5 , is found to be logically equivalent to a set of inclusions given by Kuratowski† for the set $\bar{A}$, having the properties of the closure $A+g A$ of $A$. In $\S 7$ are presented various properties of the elementary sets. All relations are established formally, though Axioms I, II are equivalent to the assumption of a neighborhood space with open sets for neighborhoods, so that all results may be had by classification of neighborhoods with respect to the distribution of the points of $A$ and $c A$. The symbols $\rightarrow$ and $\subset$ denote respectively implication and inclusion.

* See M. Fréchet, Les Espaces Abstraits, 1928; E. W. Chittenden, On general topology, Transactions of this Society, vol. 31 (1929), pp. 290-321; W. Sierpinski, La notion de derivée comme base d'une théorie des ensembles abstraits, Mathematische Annalen, vol. 97 (1926), pp. 321-337.

$\dagger$ C. Kuratowski, Sur l'opération $\bar{A}$ de l'analysis situs, Fundamenta Mathematica, vol. 3 (1922), pp. 182-199. 
2. Reduction Formulas. From the distributive law I we have $g A+g c A=g S$. Taking complements, we obtain the following lemma.

Lemмa 1. We have $\operatorname{cg} A \subset g c A+J$, where $J=\operatorname{cgS}$, the set of isolated points of the space.

From I also follows the monotonic property

$$
X \subset Y \rightarrow g X \subset g Y .
$$

Since $X \subset Y \rightarrow c Y \subset c X$, we have also, by (1),

$$
\begin{aligned}
X \subset Y & \rightarrow g c Y \subset g c X, \\
& \rightarrow c g Y \subset c g X, \\
& \rightarrow g c g Y \subset g c g X .
\end{aligned}
$$

Instead of referring to the monotonic property (1), it will be frequently convenient to speak of operating with $g$ on $X \subset Y$. Likewise we shall operate with $c, g c, \cdots$.

Lemma 2. For any operator $\phi, J \subset \operatorname{cg} \phi A$.

The lemma follows on applying the operator $c g$ to the inclu$\operatorname{sion} \phi A \subset S$.

LemMA 3. For any operator $\phi, g J \subset g c g \phi A$.

This is an immediate consequence of (1) and Lemma 2.

The following reduction formulas hold for any ordinal $\alpha$.

Formula I. $g c g A=g c g^{\alpha} A$. Operating with $g$ on Lemma 1 , we obtain by the closure property II,

$$
g c g A \subset g^{2} c A+g J \subset g c A+g J,
$$

whence, on replacing $A$ by $g A$, we find $g c g^{2} A \subset g c g A+g J$; or by Lemma $3, g c g^{2} A \subset g c g A$. The reversed inclusion is obtained by operating with $g c$ on the inclusion $g^{2} A \subset g A$, so that we shall have $g c g A=g c g^{2} A$. Replacing $A$ by $g A$, we get $g c g A=g c g^{3} A$, and the desired relation follows at once.

Formula II. $g c g A=g^{\alpha} c g A+g J$. Replacing $A$ by $c g A$ in $g^{2} A \subset g A$, we shall have $g^{2} c g A \subset g c g A$, whence, by Lemma 3,

$$
g^{2} c g A+g J \subset g c g A .
$$


Replacing $A$ by $g A$ in (2), we get $g c g^{2} A \subset g^{2} c g A+g J$, whence, by Formula I, $g c g A \subset g^{2} c g A+g J$. From this and (3) follows

$$
g c g A=g^{2} c g A+g J .
$$

Operating on (4) with $g$, we have $g^{2} c g A=g^{3} c g A+g^{2} J$, whence (4) may be written in the form $g c g A=g^{3} c g A+g J$. The required formula follows immediately.

Preliminary to Formula III we have the following lemma.

Lemma 4. $g X c g c Y \subset g(X Y) \subset g X g Y$.

The second inclusion is a direct consequence of the monotonic property. As for the first, we may write, by I,

whence

$$
g X=g(X Y+X c Y)=g(X Y)+g(X c Y),
$$

(5) $g X c g c Y=[g(X Y)+g(X c Y)] c g c Y \subset g(X Y)+g(X c Y) c g c Y$;

but, by the second inclusion of the lemma, $g(X c Y) \subset g X g c Y$, so that we have $g(X c Y) \operatorname{cgc} Y \subset g X g c Y c g c Y=0$, and, by (5), the lemma is established.

Formula III. $g c g A=\operatorname{cog} \operatorname{cgcg} A$. In Lemma 1 we replace $A$ by $\operatorname{cg} A$, obtaining, by II, $\operatorname{cgcg} A \subset g^{2} A+J \subset g A+J$, whence, on operating by $g$,

$$
g \operatorname{cgc} g A \subset g^{2} A+g J \subset g A+g J .
$$

Again, replacing $A$ by $\operatorname{cg} A$, we have, by Lemma 3,

$$
\operatorname{gcg} \operatorname{cgcg} A \subset g c g A+g J=\operatorname{gcg} A \text {. }
$$

To establish the reversed inclusion, we proceed as follows. Operating on (6) with $g c$, we have

$$
g(\operatorname{cg} A \operatorname{cg} J) \subset g c g c g c g A .
$$

Now in Lemma 4 , on setting $X=c g A, Y=c g J$, we shall have $g c g A c g^{2} J \subset g(c g A c g J)$, or by the closure property,

$$
g c g A \subset g(c g A c g J)+g J .
$$

But, by Lemma 3, $g J \subset g(c g A c g J)$, so that (8) reduces to

$$
g c g A \subset g(c g A c g J) \text {. }
$$

By (7) and (9) the desired relation is established. 
3. A Canonical System of Sets. Consider now a set $\phi A$, where $\phi$ is any combination of the deriving and complementing operations. By Formulas I, III, we have

$$
g^{\alpha} c g^{\beta} c g^{\gamma} c g^{\delta} A=g^{\alpha} \operatorname{cgcgc} g A=g^{\alpha} c g A .
$$

Thus it appears that when any derived set of $A$ is operated on with $g c$, the only distinct sets obtainable by further operations with $g$ and $c$ are of the types $g^{\alpha} c g A, c g^{\alpha} c g A, g^{\alpha} c g c g A, c g^{\alpha} c g c g A$. The following theorem is thus established.

Theorem 1. For any operator $\phi$, the set $\phi A$ reduces to $A, c A$, $\operatorname{cg} A, \operatorname{cgc} A, \operatorname{cgcg} A, \operatorname{cgcgc} A$, or else may be obtained by operating on one of these sets with $g^{\alpha}$ or $c g^{\alpha}$.

4. Definition of the Elementary Sets. The following lemma is needed.

Lemma 5. For any ordinals $\alpha, \beta$, and operator $\phi$,

$$
\begin{aligned}
g^{\alpha} c g \phi A & =g^{\beta} c g \phi A c g J+g^{\alpha} c g \phi A g J, \\
c g^{\alpha} c g \phi A & =c g^{\beta} c g \phi A c g J+c g^{\alpha} c g \phi A g J .
\end{aligned}
$$

By the closure property and the Formula II previously stated, we have

$$
g^{\alpha} c g \phi A \subset g c g \phi A=g^{\beta} c g \phi A+g J,
$$

whence we obtain $g^{\alpha} c g \phi A c g J=g^{\beta} c g \phi A c g J$, and

$$
g^{\alpha} c g \phi A=g^{\alpha} c g \phi A c g J+g^{\alpha} c g \phi A g J=g^{\beta} c g \phi A c g J+g^{\alpha} c g \phi A g J .
$$

The rest of the lemma follows on taking complements.

The following fundamental inclusions hold for all values of $\alpha$. I1.

$$
c g^{\alpha} c A c \bar{J} \subset \operatorname{cgcgcgc} A c J \text {. }
$$

Operating on (6) with $c g^{\alpha-1}$, and replacing $A$ by $c A$, we have $c g^{\alpha} c A c g J \subset c g^{\alpha} c g c g c A$, whence $c g^{\alpha} c A c g J \subset c g^{\alpha} c g c g c A c g J$. But, choosing $\beta=1$ in Lemma 5 , we have $c g^{\alpha} \operatorname{cgcgcAcgJ}=\operatorname{cgcgcgcAcgJ}$, so that the preceding inclusion becomes, by Lemma 3,

$$
c g^{\alpha} c A c g J \subset \operatorname{cgcgcgc} A c g J=\operatorname{cgcgcgc} A .
$$

Multiplying by $c J$, we have $c g^{\alpha} c A c \bar{J}=c g^{\alpha} c A c g J \subset \operatorname{cgcgcgcA} c J$, which is the desired inclusion.

$$
\operatorname{cgcgcgcAcJ} \subset g^{\alpha} \operatorname{cgc} A \text {. }
$$

In Lemma 1 we replace $A$ by $\operatorname{cgcgc} A$, and have, by II, 


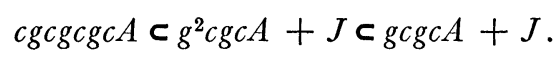

Multiplying by $c g J c J$, we obtain $c g c g c g c A c J \subset g c g c A c g J c J$, by Lemma 3. But by Formula II, $g c g c A c g J=g^{\alpha} c g c A c g J$, whence the above inclusion yields $c g c g c g c A c J \subset g^{\alpha} c g c A c g J c J \subset g^{\alpha} c g c A$.

I3.

$$
\operatorname{cgcgcgcAcJ} \subset \operatorname{cg}{ }^{\alpha} \operatorname{cg} A \text {. }
$$

Applying the operator $g c$ to (2), we get $g(\operatorname{cgcAcgJ}) \subset \operatorname{gcgcg} A$. Since, by (9), $g c g c A \subset g(c g c A c g J)$, we have then $g c g c A \subset g c g c g A$, or, replacing $A$ by $c A, g c g A \subset g c g c g c A \subset g c g c g c A+J$. From the closure of derived sets, we have $g^{\alpha} c g A \subset g c g A \subset g c g c g c A+J$. On taking complements, the desired inclusion is obtained.

By operating with $c$ on $\mathrm{I} 3, \mathrm{I} 2, \mathrm{I} 1$, respectively, and replacing $A$ by $c A$, the following inclusions are obtained:

$$
g^{\alpha} \operatorname{cgc} A \subset \operatorname{gcgcg} A+J .
$$

I5.

$$
\operatorname{cg}^{\alpha} \operatorname{cg} A \subset \operatorname{gcg} \operatorname{cg} A+J .
$$

I6.

$$
\operatorname{gcg} c g A+J \subset g^{\alpha} A+\bar{J} .
$$

Consider now the following special forms of the inclusions I1-I6, where $\alpha_{1}$ and $\alpha_{2}$ are the least ordinals $\alpha$ such that $g^{\alpha} c g c A$ and $g^{\alpha} c g A$, respectively, are perfect:

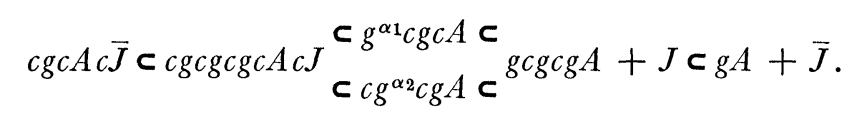

From the monotonic nature of the six sets (10), it is seen that the first, the complement of the sixth, and the differences of the sixth and fifth, the fifth and second, and the second and first, are mutually disjoined and fill the space $S$. The difference of the fifth and second sets, namely, $(\operatorname{gcg} c g A+J)(\operatorname{gcg} \operatorname{cgc} A+J)$, may be decomposed into four non-overlapping sets by means of the intervening sets, $g^{\alpha_{1}} c g c A$ and $c g^{\alpha_{2}} c g A$. Thus we obtain a decomposition of $S$ into eight non-overlapping sets. With certain reductions by (10), Lemmas 2 and 3, we list these eight sets, and shall refer to them as elementary or, more briefly, $E$ sets:

$$
\begin{aligned}
& E_{1}=c g c A c \bar{J}, \quad E_{2}=c g c g c g c A c J c(c g c A c \bar{J})=c g c g c g c A g c A, \\
& E_{3}=g^{\alpha_{1}} c g c A c g^{\alpha_{2}} c g A c(c g c g c g c A c J)=g^{\alpha_{1}} c g c A c g^{\alpha_{2}} c g A g c g c g c A, \\
& E_{4}=c g A c \bar{J}, \quad E_{5}=(g A+\bar{J}) c(g c g c g A+J)=g A c g c g c g A, \\
& E_{6}=(g c g c g A+J) c g^{\alpha_{1}} c g c A c\left(c g^{\alpha_{2}} c g A\right)=g c g c g A c g^{\alpha_{1}} c g c A g^{\alpha_{2}} c g A \\
& E_{7}=g^{\alpha_{1}} c g c A g^{\alpha_{2}} c g A, \quad E_{8}=c g^{\alpha_{1}} c g c A c g^{\alpha_{2}} c g A .
\end{aligned}
$$


It is seen that the replacement of $A$ by $c A$ interchanges $E_{1}$ and $E_{4}, E_{2}$ and $E_{5}, E_{3}$ and $E_{6}$. Thus, corresponding to a theorem holding for a set of one of these pairs, there is an identical theorem valid for the other set. We shall make use of this symmetry.

5. The General Canonical System.

Lemma 6. $J \subset E_{8} ; g J \subset E_{3}+E_{6}+E_{7}+E_{8}$.

It is seen by (11) that every $E$ set except $E_{8}$ has either the factor $c J$ or else a factor $g \phi A$, and so by Lemma 2 is disjoined from $J$. Likewise $E_{1}$ and $E_{4}$ contain the explicit factor $c g J$, while $E_{2}$ and $E_{5}$ have factors $\operatorname{cgcgcgc} A$ and $\operatorname{cgcgcg} A$, which by Lemma 3 are contained in $c g J$.

By Lemma 6, 3, and 5, we may obtain from (11) the following decomposition of the sets mentioned in Theorem 1.

$$
\begin{aligned}
g^{\alpha} c g A & =E_{4}+E_{5}+c g J\left(E_{6}+E_{7}\right)+g^{\alpha} c g A g J . \\
c g^{\alpha} c g A & =E_{1}+E_{2}+c g J\left(E_{3}+E_{8}\right)+c g^{\alpha} c g A g J . \\
g^{\alpha} c g c A & =E_{1}+E_{2}+c g J\left(E_{3}+E_{7}\right)+g^{\alpha} c g c A g J . \\
c g^{\alpha} c g c A & =E_{4}+E_{5}+c g J\left(E_{6}+E_{8}\right)+c g^{\alpha} c g c A g J . \\
g^{\alpha} c g c g A & =E_{1}+E_{2}+c g J\left(E_{3}+E_{6}+E_{7}\right)+c \bar{J} E_{8}+g^{\alpha} c g c g A g J . \\
c g^{\alpha} c g c g A & =E_{4}+E_{5}+J+c g^{\alpha} c g c g A g J . \\
g^{\alpha} c g c g c A & =E_{4}+E_{5}+c g J\left(E_{3}+E_{6}+E_{7}\right)+c \bar{J} E_{8}+g^{\alpha} c g c g c A g J . \\
c g^{\alpha} c g c g c A & =E_{1}+E_{2}+J+c g^{\alpha} c g c g c A g J .
\end{aligned}
$$

Expressions for the sets $g^{\alpha} A, c g^{\alpha} A, g^{\alpha} c A, c g^{\alpha} c A$ of Theorem 1 in terms of the $E$ sets cannot be obtained in this manner, since Lemma 5 does not apply to these sets. We have instead the following lemma.

\section{LEMMA 7.}

$$
\begin{aligned}
& P_{1} c g J=E_{1}+E_{2}+c g J\left(E_{3}+E_{6}+E_{7}\right)+c \bar{J} E_{8}+P_{1} E_{5}, \\
& P_{2} c g J=P_{2} E_{2}+c g J E_{3}+E_{4}+E_{5}+c g J\left(E_{6}+E_{7}\right)+c \bar{J} E_{8},
\end{aligned}
$$

where $P_{1}, P_{2}$, are respectively the perfect components of $A, c A$.

By (11), Lemma 6, and I6, we have for every $\alpha$

$$
E_{1}+E_{2}+c g J\left(E_{3}+E_{6}+E_{7}\right)+c \bar{J} E_{8}=\operatorname{gcgcg} A c \bar{J} \subset g^{\alpha} A \text {. }
$$

Since $E_{4}$ is disjoined from $g A$, the lemma follows. 
By (11) and Lemma 7 we have

$$
\begin{aligned}
g^{\alpha} A= & E_{1}+E_{2}+c g J E_{3}+P_{1} E_{5}+\sum_{\beta \geqq \alpha}\left(g^{\beta} A-g^{\beta+1} A\right) \\
& +c g J\left(E_{6}+E_{7}\right)+c \bar{J} E_{8}+P_{1} g J \\
c g^{\alpha} A= & E_{4}+\sum_{\beta<\alpha}\left(g^{\beta} A-g^{\beta+1} A\right)+J+g J c g^{\alpha} A, \\
g^{\alpha} c A= & P_{2} E_{2}+\sum_{\beta \geqq \alpha}\left(g^{\beta} c A-g^{\beta+1} c A\right)+c g J E_{3}+E_{4}+E_{5} \\
& +c g J\left(E_{6}+E_{7}\right)+c \bar{J} E_{8}+P_{2} g J \\
c g^{\alpha} c A= & E_{1}+\sum_{\beta<\alpha}\left(g^{\beta} c A-g^{\beta+1} c A\right)+J+g J c g^{\alpha} c A .
\end{aligned}
$$

In (12) and (14) we have the required decomposition of the sets of Theorem 1 . Since by (13) each set $g^{\beta} A-g^{\beta+1} A$ lies in $E_{5}+g J$, we may sum up our results in the following theorem.

Theorem 2. Any finite sum of finite products $\Sigma \Pi \phi A$ is expressible, aside from a subset of $g J$, as a sum chosen from $E_{1}$, $P_{2} E_{2}, g^{\beta} c A-g^{\beta+1} c A, c g J E_{3}, E_{4}, P_{1} E_{5}, g^{\beta} A-g^{\beta+1} A, c g J E_{6}, c g J E_{7}$, $c \bar{J} E_{8}, J$, and products of these sets by $A$ and $c A$.

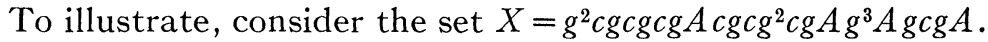
Applying Formulas I and III, we have $X=g^{2} c g A c g c g c g A g^{3} A g c g A$, whence by the closure of derived sets, $X=g^{2} \operatorname{cg} A \operatorname{cgcgcg} A g^{3} A$. Finally, (12) and (14) give $X=P_{1} E_{5}+\sum_{\beta \geqq 3}\left(g^{\beta} A-g^{\beta+1} A\right)$, aside from a subset of $g J$.

6. Specializations. Most of the spaces commonly occurring in geometry and analysis, such as Euclidean $n$-space, Hilbert space, and all continua, have the self-dense property, and also the property $g 0=0$. If we set $J=g J=0$, the results of the preceding sections are considerably simplified. Formula II reduces to $\operatorname{gcg} A=g^{\alpha} \operatorname{cg} A$, and the inclusions (10) become*

$$
\operatorname{cgcA\subset \operatorname {cgcgcgc}A} \begin{aligned}
& \subset \operatorname{gcgc} A \subset \\
& \subset \operatorname{cgcg} A \subset \operatorname{gcgcg} A \subset g A .
\end{aligned}
$$

The elementary sets (11) reduce to

$$
\text { (16) } \begin{aligned}
E_{4} & =c g A, \quad E_{5}=g A \operatorname{cgcgcg} A, \quad E_{6}=\operatorname{gcgcg} A g c g A c g c g c A, \\
E_{7} & =g c g c A g c g A, \quad E_{8}=\operatorname{cgcgcAcgcg} A,
\end{aligned}
$$

* These inclusions are given by Kuratowski, loc. cit., for the closure function. 
while the general canonical system of $\$ 5$ reduces to

$$
\begin{aligned}
E_{1}, \quad P_{2} E_{2}, \quad & \sum\left(g^{\beta} c A-g^{\beta+1} c A\right), \quad E_{3}, \quad E_{4}, \quad P_{1} E_{5}, \\
& \sum\left(g^{\beta} A-g^{\beta+1} A\right), \quad E_{6}, \quad E_{7}, \quad E_{8} .
\end{aligned}
$$

The requirement $g J=0$ yields a space slightly more general than the preceding, including the case where isolated points $J$ exist, yet have a null derived set. The formulas of $\$ \$ 2-5$ specialize, however, to the very forms (15), (16), (17). The structure of the $E$ sets is unchanged except that $E_{8}$ includes the set $J$.

We may also specialize the formulas of $\$ \S 2-5$ by requiring $J=0$, thus including the case $J=g J=0$ and also the case where the derived set of the null set is not null. By the monotonic property we have for every set $A, g 0=g J \subset g A$, so that the inclusions (10) again reduce to (15), and the definitions (16) hold without change. Since $g J \subset g A$, we see that $E_{7}$ includes $g J$.

If we require $J+g J=S$, the canonical system of Theorem 2 is considerably simplified, since the inclusions (10) reduce to

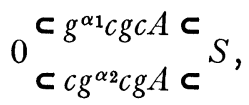

so that the only non-null $E$ sets are $E_{3}, E_{6}, E_{7}, E_{8}$.

7. The Nature of the Elementary Sets. The following theorems may be obtained.

Theorem 3. $E_{1}+E_{4}+E_{8}+g J$ is dense on $g S$.

ThEOREM 4. $E_{3} c g J=g E_{1} c g E_{4} g E_{8} c g J$.

Theorem 5. $E_{2}=c g J g E_{1} c g E_{4} c g E_{8} g c A$.

Theorem 6. $g E_{3} \subset E_{3}+E_{7}$.

TheOREM 7. $g E_{2} \subset E_{2}+E_{3}+E_{7}+g J$.

Theorem 8. $g E_{8} \subset E_{3}+E_{6}+E_{7}+E_{8}$.

In a space $J=g J=0$ it is evident that $E_{8}=c g E_{1} c g E_{4}$, and $E_{7}=g E_{1} g E_{4}$. If the space is also of $n$ dimensions, then it follows from Theorem 3 that $E_{1}, E_{4}$, and $E_{8}$ are $n$-dimensional or else null, whereas the remaining $E$ sets are at most $(n-1)$-dimensional. Specializing further, we have the following result.

THEOREм 9. In a Euclidean space of more than one dimension, $E_{7} \subset g E_{7}+g E_{3} g E_{6}$.

St. JOSEPH, Missouri 